\title{
Ventriculoperitoneal shunt-associated abdominal cerebrospinal fluid pseudocysts and the role of laparoscopy and a proposed management algorithm in its treatment
}

$A$ report of 2 cases

Ghaida M. Fatani, MBBS, Nasir M. Bustangi, DES, DESC, Jamal S. Kamal, FRCSI, FACS, Abdulrahman J. Sabbagh, MBChB, FRCSC.

\begin{abstract}
الكيس البطني الكاذب المحتوي على السائل النخاعي من المضاعفات

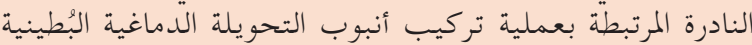

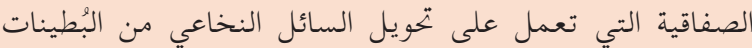

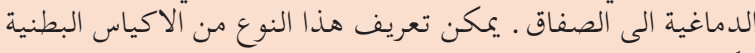

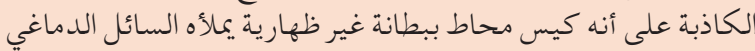

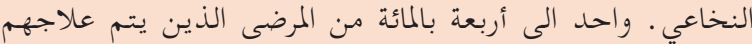

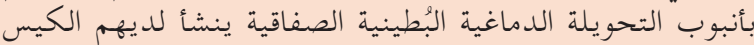

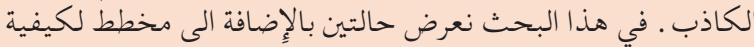

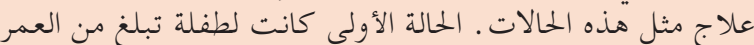

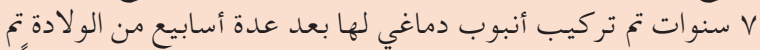

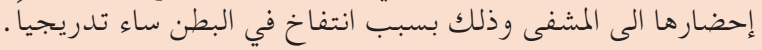

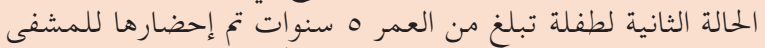

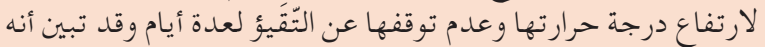

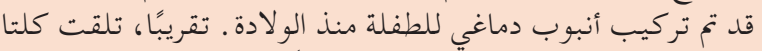

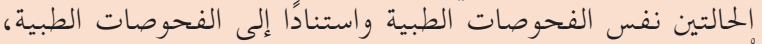

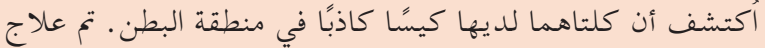

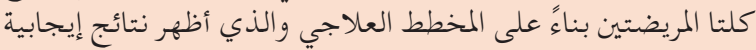

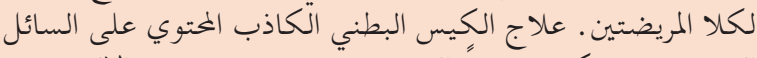

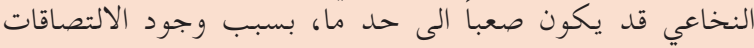

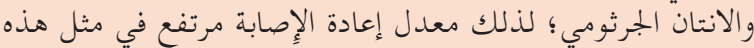
الحالات.
\end{abstract}

Abdominal cerebrospinal fluid pseudocyst (APC) is a rare complication associated with a ventriculoperitoneal shunt (VPS) placement. Abdominal cerebrospinal fluid pseudocyst is defined as a cyst surrounded by a nonepithelial lining and filled with the cerebrospinal fluid (CSF). In general, $1 \%-4.5 \%$ of all patients treated with a VPS develop CSF pseudocysts. Here, we have presented 2 cases of APC complicating VPS placement and a proposed treatment management algorithm. The cases pertained to 7 -year-old and 5-year-old children who had undergone VPS placement during infancy and presented with progressive abdominal distention and vomiting and fever, respectively. Both cases were assessed using similar investigations and imaging modalities and diagnosed with APC. However, the treatments were individually tailored based on the algorithm; successful outcomes were achieved in both patients. Abdominal cerebrospinal fluid pseudocyst treatment is difficult considering the presence of adhesions and infection and is associated with a high recurrence rate.

Neurosciences 2020; Vol. 25 (4): 320-326 doi: 10.17712/nsj.2020.4.20200053

From the Faculty of Medicine (Fatani); from the Division of Pediatric Surgery (Bustangi, Kamal), and from the Division of Neurosurgery (Sabbagh), King Abdulaziz University, Jeddah, Kingdom of Saudi Arabia.

Received 6th October 2019. Accepted 15th April 2020.

Address correspondence and reprint request to: Dr. Ghaida M. Fatani, Faculty of Medicine, King Abdulaziz University, Jeddah, Kingdom of Saudi Arabia.E-mail: Ghaida.f95@gmail.com

ORCID ID: https://orcid.org/0000-0001-5101-6764

$\mathrm{V}$ entriculoperitoneal shunt (VPS) placement is one of the most common cerebrospinal fluid (CSF) diversion procedures performed by neurosurgeons globally. It is the most commonly used procedure among surgeons with different levels of expertise in managing hydrocephalus of various etiologies. In this procedure, excess CSF is diverted from the lateral ventricles to the peritoneum. ${ }^{1}$ Less commonly, the

Disclosure. Authors have no conflict of interests, and the work was not supported or funded by any drug company. 
CSF may be diverted to other sites such as the right atrium, and to a much lesser degree, to the gallbladder, pleural space, and ureter. However, because of the lower rates of serious complications, it is considered advantageous to divert the CSF into the peritoneal cavity. ${ }^{2}$ Ventriculoperitoneal shunt placement has been associated with several complications, such as infection, VPS malfunction, and intestinal perforation as well as abdominal pseudocyst formation. ${ }^{3}$ Abdominal CSF pseudocyst (APC) is defined as a cyst surrounded by a lining that is devoid of epithelial cells and is filled with the CSF. ${ }^{4}$ Abdominal CSF pseudocyst formation is of unknown etiology and its incidence is $1 \%$ to $4.5 \% .^{5}$ Here, we report 2 pediatric cases of APC that were treated using the treatment management algorithm proposed in this report.

Case Report. Patient 1. Informed consent was obtained from the family. The timeline for this case is summarized in Figure 1. This case pertained to a 7-year-old girl, who presented with a 2-month history of progressive abdominal distention, headaches, and decreased oral intake. She was born preterm as one of a triplet with a history of meningitis, which led to postmeningitic hydrocephalus that required VPS placement. She was followed up by the neurosurgical department for 3 years without undergoing shunt revisions or facing any complications. She was then lost to follow-up until she presented with new symptoms.

Clinical findings. On examination, she appeared pale. Her abdomen was distended with an everted umbilicus and prominent veins. Fluid thrill was positive and neurological examination, including fundoscopy, revealed normal results.

Diagnostic assessment. The results of basic investigations, CSF analysis, and microbial cultures are presented in Table 1. Abdominal radiography showed a paucity of gas in the central bowel along with peripheral displacement of the bowel (Figure 2A). A contrastenhanced computed tomography (CT) of the abdomen and pelvis revealed the presence of a giant "CSF-oma" occupying the entire peritoneal cavity and pushing the bowel loops laterally. The VPS catheter was visualized within the abdomen and found to have a normal course (Figure 2B). An axial CT of the brain showed the shunt tip within the lateral ventricles along with a decreased sulcal and gyral pattern scalloping the inside of the calvarium. We hypothesized that a retrograde buildup due to increased intra-abdominal pressure may have affected the proximal shunt function (Figure 2C).

Mesenteric cysts, pancreatic pseudocysts, cystic teratomas, simple or complicated ovarian cysts, and abdominal CSF pseudocysts were considered in the differential diagnosis. However, the patient's presentation was more coherent with the diagnosis of an abdominal CSF pseudocyst.

Therapeutic intervention. We performed an

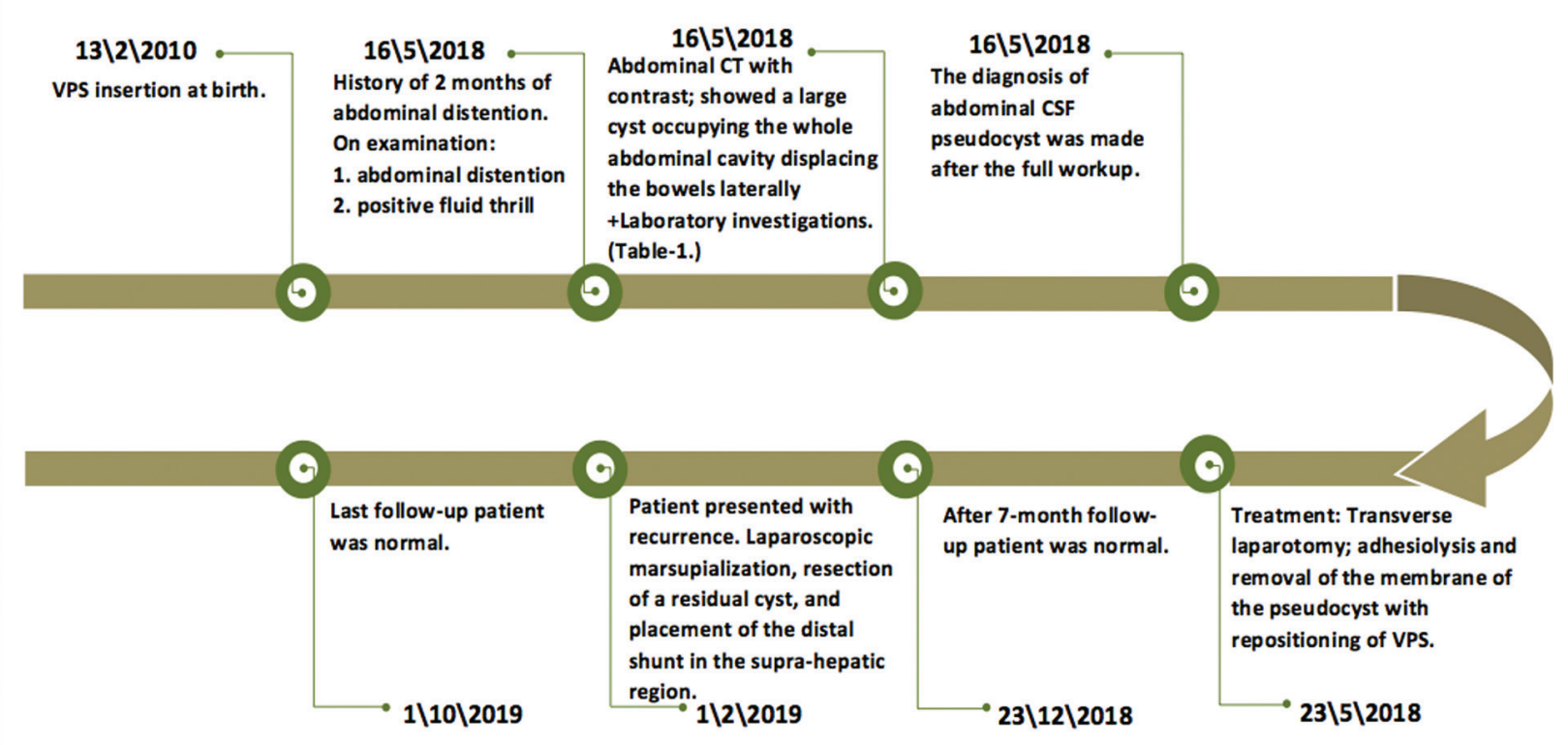

Figure 1 - Timeline of the first case. The patient shunted since infancy, presented later with abdominal distension, headache and decrease oral intake. Repositioning of ventriculoperitoneal shunt (VPS) to virgin area in the abdomen. CSF: cerebrospinal fluid 


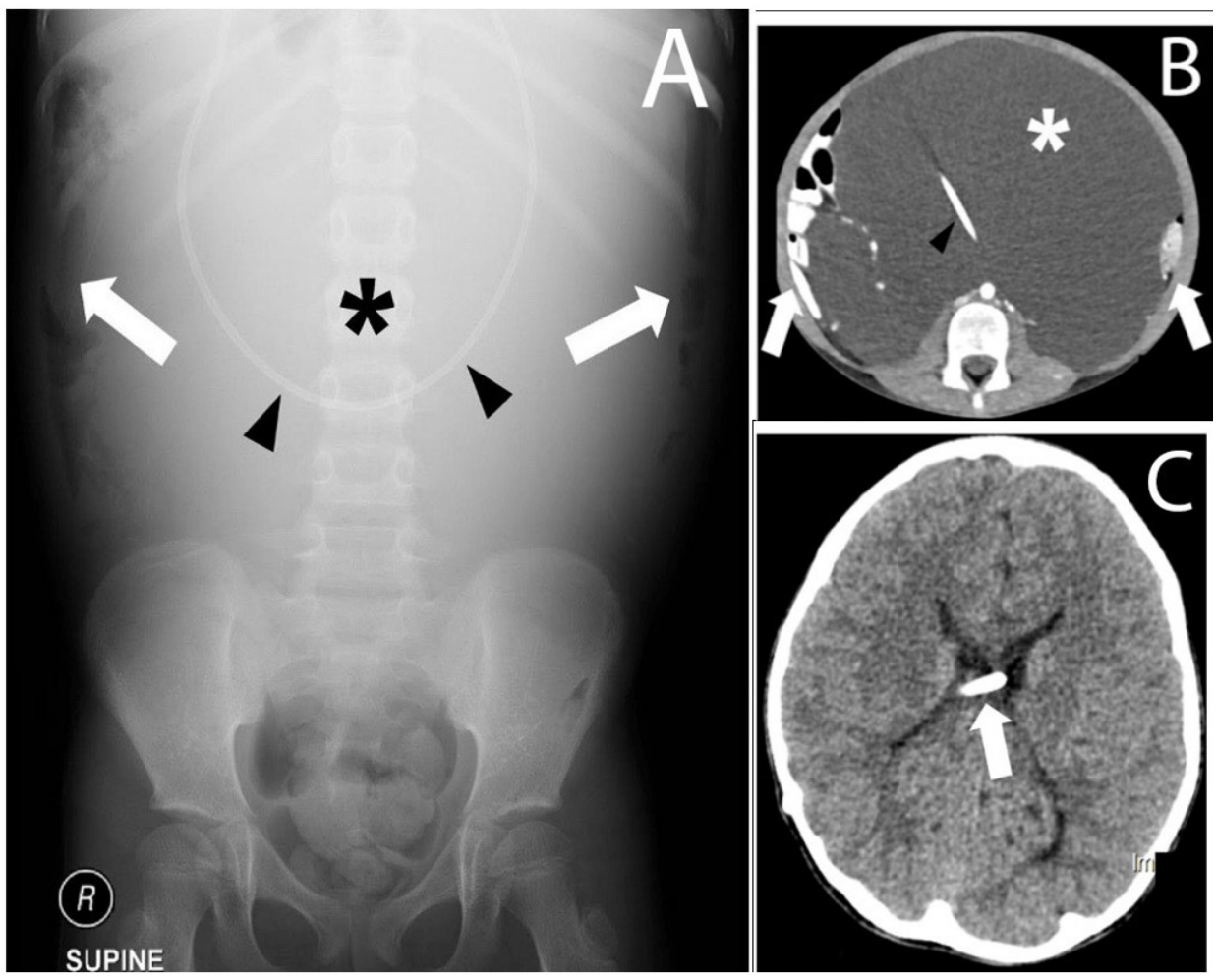

Figure 2 - Seven-year-old female with progressive abdominal distension. A) Frontal radiograph showing large mid-abdominal opacity (asterisk) displacing the air-filled bowel laterally (arrows). Part of the shunt is in the mid abdomen (arrowhead). B) Axial enhanced computed tomography showing a large loculated fluid (asterisk) displacing the bowels laterally (arrows). The shunt is seen within the fluid (arrowhead). C) Axial unenhanced computed tomography showing intraventricular location of the shunt (arrow) with no hydrocephalus.
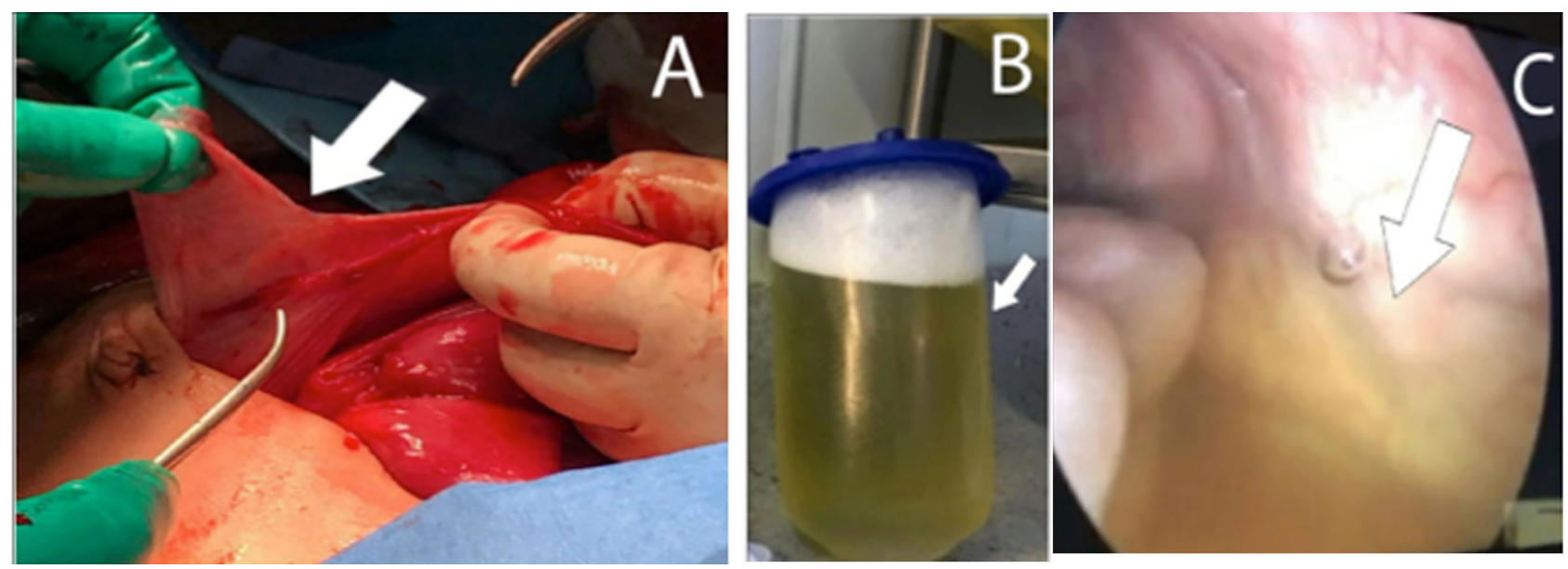

Figure 3 - Intraoperative images for first case. A) walls of the pseudocyst (arrowhead). B) The collected fluid evacuated from the pseudocyst (indicated by the arrow) C) Laparoscopic view from inside the cyst with remaining fluid after aspiration (arrowhead).

exploratory laparoscopic procedure via the umbilical port. The large CSF-filled pseudo-cyst was evacuated. A sample was sent for culture and cytological analysis, which revealed clusters of reactive mesothelial cells, lymphocytes, neutrophils, and histocytes, with negative bacterial culture. Because the pseudo-cyst was large enough to occupy nearly the entire peritoneal cavity, there were no possible sites for another trocar insertion; therefore, the procedure was converted from 
a laparoscopic exploration to transverse laparotomy. Adhesiolysis was performed by deroofing the cyst walls to avoid compromising the bowel walls, and the VPS catheter was repositioned to a new area in the abdomen. The post-operative course was uneventful (Figure 3.)

Follow-up and outcomes. The patient was followed up regularly by both neurosurgical and pediatric surgical clinics without complications. She returned 8 months after the surgery to the emergency department with a recurrence. The CSF was drained and laparoscopic

Table 1 - Laboratory results for the first case.

\begin{tabular}{lcc}
\hline Laboratory & Results & Normal range \\
\hline White blood counts & $8.66 \mathrm{~K} \mathrm{U} / \mathrm{L}$ & $4.5-11.5 \mathrm{~K} \mathrm{U} / \mathrm{L}$ \\
Aspartate transaminase & $17 \mathrm{U} / \mathrm{L}$ & $15-37 \mathrm{U} / \mathrm{L}$ \\
Alanine aminotransferase & $16 \mathrm{U} / \mathrm{L}$ & $12-78 \mathrm{U} / \mathrm{L}$ \\
Alkaline phosphatase & $160 \mathrm{U} / \mathrm{L}$ & $54-128 \mathrm{U} / \mathrm{L}$ \\
Bilirubin - total & $4.0 \mathrm{~g} / \mathrm{L}$ & $0-17 \mathrm{~g} / \mathrm{L}$ \\
Albumin & $30 \mathrm{~g} / \mathrm{L}$ & $40.2-47.6 \mathrm{~g} / \mathrm{L}$ \\
Cerebrospinal fluid protein & $15.06 \mathrm{~g} / \mathrm{L}$ (high) & $0.2-0.4 \mathrm{~g} / \mathrm{L}$ \\
Cerebrospinal fluid glucose & $4.6 \mathrm{mmol} / \mathrm{L}$ (high) & $2.3-4.1 \mathrm{mmol} / \mathrm{L}$ \\
Cerebrospinal fluid bacterial \& & $\mathrm{Negative}$ & \\
fungal culture & & \\
C-reactive protein & $11.5 \mathrm{mg} / \mathrm{L}$ (High) & $0-3 \mathrm{mg} / \mathrm{L}$ \\
\hline
\end{tabular}

pseudo-cyst marsupialization, resection of a residual cyst, and placement of the distal shunt in the suprahepatic region was performed. Her condition remained normal until her last follow-up at 8 months.

Patient 2. Informed consent was obtained from the family. The timeline for this case is summarized in Figure 4. This case pertained to a 5-year-old girl with paraplegic myelomeningocele (MMC) who had undergone shunt placement for hydrocephalus shortly after MMC closure at birth. At 3 weeks of age, she underwent multiple shunt revisions and remained healthy until the current presentation. The patient presented to the emergency department with 2-day history fever and vomiting.

Clinical findings. On examination, the abdomen was distended and tense.

Diagnostic assessment. A contrast-enhanced CT of the abdomen and pelvis revealed a large APC with multiple septations occupying the peritoneal cavity (Figure 5). The differential diagnosis was the same as that in the first case report.

Therapeutic intervention. Laparoscopic exploration revealed large amounts of turbid CSF and pus from the cyst. The peritoneum was aggressively washed using the laparoscopic approach with externalization of the distal shunt tip. The drained fluid tested positive for the presence of Enterococcus faecium (Table 2), and cytological examinations confirmed reactive mesothelial

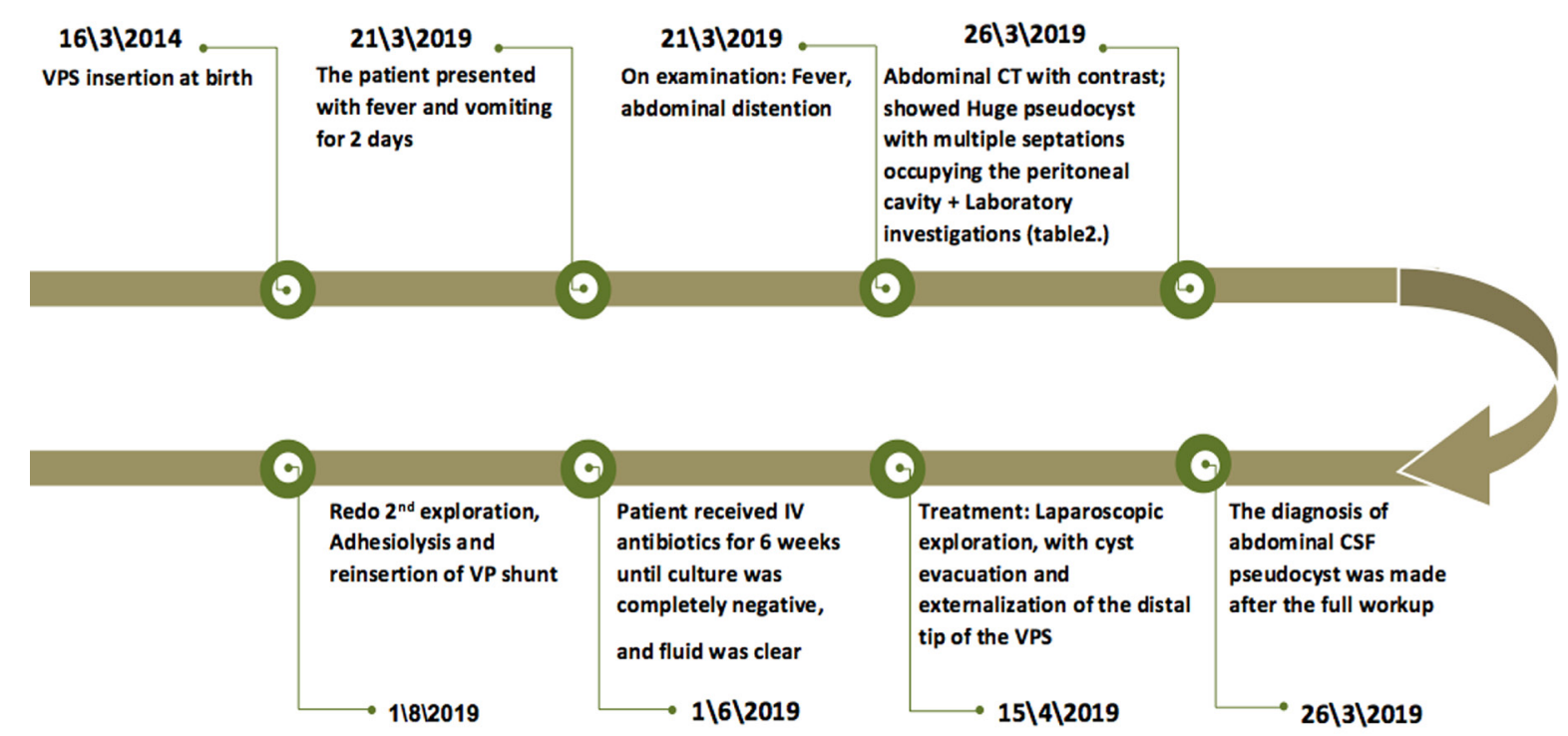

Figure 4 - Timeline of the second case. Five years old child shunted since birth, presented with fever and vomiting for 2 days. Laparoscopic externalization of the distal tip of ventriculoperitoneal shunt (VPS). 

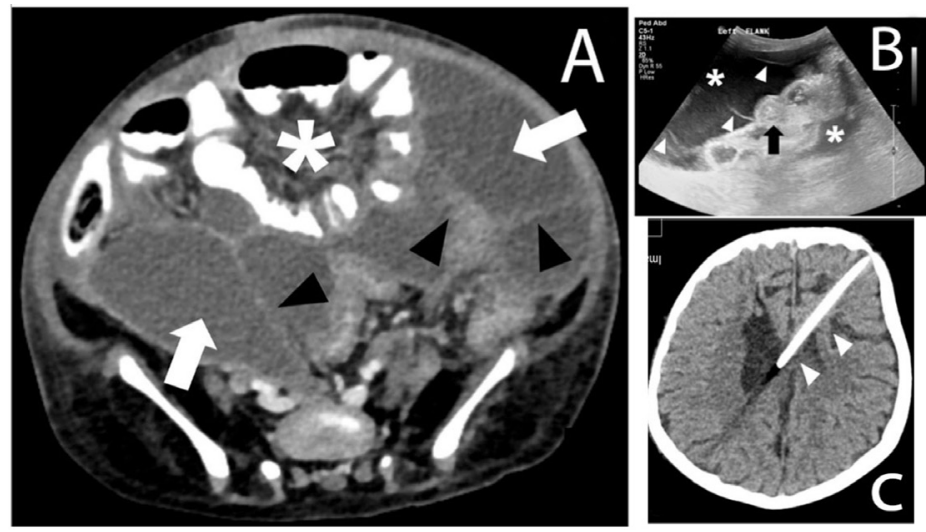

Figure 5 - Five-year-old female with fever and vomiting. A) Axial enhanced computed tomography showing complex fluid collections (arrows) with multiple septa (arrowheads) displacing the bowel loops (asterisk). B) Ultrasound image of the left flank showing complex fluid (asterisks) with multiple septa (arrowheads) surrounding the collapsed bowel loops (arrow). C) Axial unenhanced computed tomography showing intraventricular location of the shunt (arrow) with no hydrocephalus.

Table 2 - Laboratory Results for the second case.

\begin{tabular}{lcc}
\hline Laboratory & Results & Normal range \\
\hline White blood counts & $17 \mathrm{~K} \mathrm{U} / \mathrm{L}$ & $4.5-11.5 \mathrm{~K} \mathrm{U} / \mathrm{L}$ \\
Aspartate transaminase & $339 \mathrm{U} / \mathrm{L}$ & $15-37 \mathrm{U} / \mathrm{L}$ \\
Alanine aminotransferase & $215 \mathrm{U} / \mathrm{L}$ & $12-78 \mathrm{U} / \mathrm{L}$ \\
Alkaline phosphatase & $138 \mathrm{U} / \mathrm{L}$ & $54-128 \mathrm{U} / \mathrm{L}$ \\
Bilirubin - total & $5 \mathrm{~g} / \mathrm{L}$ & $0-17 \mathrm{~g} / \mathrm{L}$ \\
Albumin & $34 \mathrm{~g} / \mathrm{L}$ & $40.2-47.6 \mathrm{~g} / \mathrm{L}$ \\
Cerebrospinal fluid protein & $0.32 \mathrm{~g} / \mathrm{L}$ & $0.2-0.4 \mathrm{~g} / \mathrm{L}$ \\
Cerebrospinal fluid glucose & $3.0 \mathrm{mmol} / \mathrm{L}$ & $2.3-4.1 \mathrm{mmol} / \mathrm{L}$ \\
Cerebrospinal fluid bacterial $\&$ & Positive & \\
fungal culture & & $0-3 \mathrm{mg} / \mathrm{L}$ \\
C-reactive protein & $4.34 \mathrm{mg} / \mathrm{L}$ & \\
\hline
\end{tabular}

cells, lymphocytes, neutrophils, and histocytes. The patient was on a course of intravenous antibiotics for 6 weeks. After 8 weeks with negative cultures, a second laparoscopic exploration with adhesiolysis was followed by VPS catheter placement.

Follow-up and outcomes. The postoperative course was uneventful. She remained healthy until the last follow-up visit at 18 months.

Discussion. The pathophysiology underlying the development of APC is not well understood. It has been postulated that an inflammatory process could considerably influence the formation of CSF pseudocysts. It is possible that this could either be associated with an infection, since it plays a major role in APC formation, or a peritoneal reaction to the
VPS. Other causes, such as multiple shunt revisions, peritoneal adhesions, and elevated CSF proteins may also influence the buildup of CSF within a cyst. ${ }^{6,7}$ Forty-five reported cases of APC were found in the literature, none of which provided a definitive cause for the formation of CSF pseudocysts.

In the first case, the patient presented with a pseudocyst 7 years after the primary shunt insertion and with no history of shunt revisions. This patient had high CSF protein levels, which we hypothesize to be the cause of her APC. Another possible cause is related to an inflammatory process, attributed to the presence of the distal VPS catheter. Other etiologies are possible; therefore, determining the definitive underlying cause is improbable and would not change the management as long as it is noninfectious. The second patient presented with a purulent APC and a history of multiple shunt revisions, making infection the likely the etiology behind the development of APC.

It is imperative to study and recognize the symptoms since this is the first step toward successfully manage the disease. Furthermore, general abdominal symptoms along with the presence of a VPS should raise suspicion for APC and should be thoroughly scrutinized. ${ }^{8}$ Some authors suggested that children typically present with symptoms of increased intracranial pressure, such as nausea, vomiting, seizures, headache, or neurological changes. In contrast, adults predominantly present with abdominal symptoms, such as abdominal pain, distension, or paralytic ileus. ${ }^{6,7,9}$ APC can be successfully diagnosed through clinical findings and radiological imaging. Brain CT scans should be performed in every patient to eliminate proximal shunt dysfunction as the underlying cause. Additionally, abdominal plain 


\section{Suspected APC in VPS patient}

Abdominal UIS, Abdominal and Pelvis CT, Brain CT, Labs (WBC, CRP, ESR)

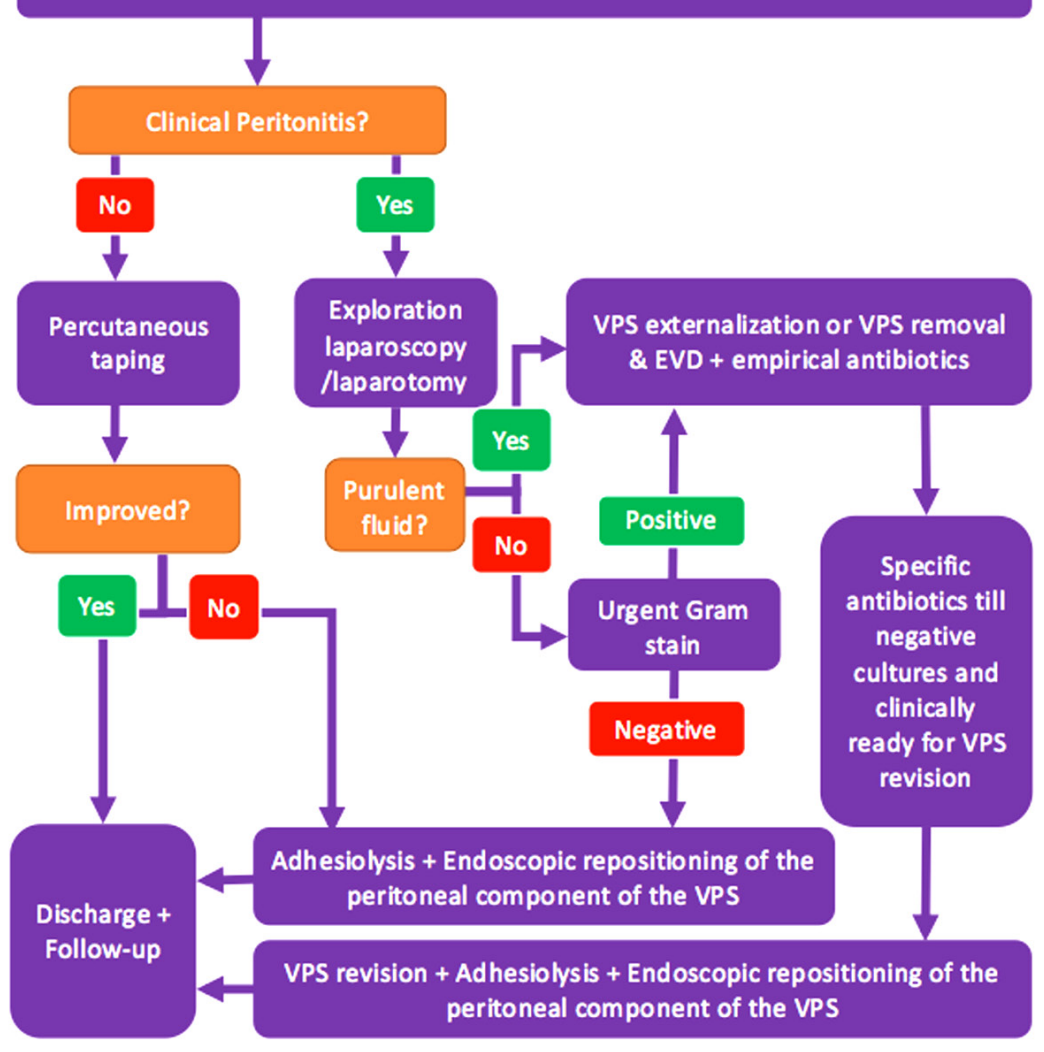

Figure 6 - An algorithm that summarizes the approach for patient with abdominal CSF pseudocyst.

radiography (as a part of shunt series), US, and/or abdominal CT should be performed subsequently to confirm the APC diagnosis. ${ }^{10}$

Figure 6 illustrates the steps that we suggest should be taken when presented with a suspected case of APC. This protocol has been followed by our center to treat APC cases, and we tailored the treatment based on the clinical signs and symptoms in patients with VPS. According to our proposed algorithm, radiological studies should be performed regardless of the presence or absence of an infection, as demonstrated in the 2 APC cases in this report.

Detection of clinical signs of peritonitis is necessary to successfully manage the condition. Patients with clinical peritonitis with a confirmed CSF pseudocyst should undergo immediate exploratory laparotomy or laparoscopy, depending on their clinical circumstances.
In patients with purulent discharge from the evacuated cyst, it is imperative to externalize the shunt or remove it and place an external ventricular drain (EVD) and administer empirical antibiotics for 6 weeks. However, if the drained fluid is not purulent there would be an urgent need to perform gram staining. In cases where the gram stain was negative, Adhesiolysis and shunt repositioning would then be performed, given that the peritoneum was suitable for repositioning. Since it has been previously suggested that $50 \%$ of patients were unable to absorb the CSF through their peritoneum, ${ }^{8}$ Some authors suggest other CSF diversion modalities, such as ventriculoarterial shunt placement, other options may include ventriculopleural shunt placement, or ventriculovesical shunt placement. ${ }^{2}$ From our experience the latter 2 are not practical.

Alternatively, if the gram stain was positive, there 
would be a need to deal with this as with purulent cyst discharge by externalizing the VPS or replacing it with EVD, followed by the protocol for the administration of empirical antibiotics as previously described.

If the patient reveals no signs and symptoms of either peritonitis or a pseudocyst, laboratory studies should be performed. If inflammatory markers such as (white blood cell) WBC and (C-reactive protein) CRP levels are elevated, the patient should be treated as a case of peritonitis. Alternatively, if there are no elevations in the levels of the typical inflammatory markers, it is recommended to proceed with percutaneous tapping as a treatment option. However, in these situations, follow-up is of paramount importance as some patients may require repeated tapping. Additionally, percutaneous drainage can be used as a tool to relieve symptoms if it precedes exploratory laparotomy or laparoscopy. ${ }^{5}$ If the patient demonstrates normal laboratory reports and no improvement with repeated percutaneous drainage, they should be treated as a case of peritonitis.

In conclusion, CSF pseudocysts are relatively rare complications of VPS. It may occur in any age group and can lead to serious complications. The fact that many patients present with abdominal symptoms in the presence of a VPS raises suspicion; accordingly, there are numerous risk factors that could be etiologically associated with APC that should be scrutinized carefully. APC treatment can be challenging considering the existence of adhesions and infection, which is further compounded by the fact that the reported recurrence rates of this condition are considerably high. Although it requires specialized skill, as well as interdisciplinary teamwork between pediatric neurosurgeons as well as pediatric surgeons. Laparoscopy plays a major role in these cases by decreasing the need for laparotomy preventing adhesions in the future.

Patient perspective. Patient 1. "According to the patient's mother, since the shunt insertion early in life, her daughter has been doing as well as her siblings until she experienced an increase in her abdominal girth. After the abdominal distension, she avoided school as she feared getting bullied by other children. She was also discouraged by her body image and the inability to put on her clothes. The mother stated that she was anxious and concerned at the time. She at times feels overwhelmed as the mother of triplets, especially as one of them became sick. This has been a significant burden on the family.

As her daughter's abdomen normalized in size following surgery, they were satisfied with the results. Furthermore, the child was back to her baseline-playful and active and returned to school.”
Patient 2. "Because of significant language barrier and difficulty accessing tertiary care, the patient's parents were very grateful for the care they finally received and stated that, as a family, they were very worried about their child. The child was very feverish and inactive for several days prior to admission to the emergency department. The mother reported feeling burdened and overwhelmed by dealing with externalized distal shunt catheters for several weeks while in the hospital especially with mobilizing the child and when cleaning and washing. To them, this was one of the worst experiences they had ever had. After recovery, they said that their child had been saved and were appreciative of their healthcare providers."

Consent was obtained from the parents of both families.

Acknowledgment. We would like to thank Dr. Rayan A. Ahyad, a pediatric radiologist from King Abdulaziz University, Jeddah, Saudi Arabia for providing assistance with radiological imaging, and Editage for English language editing.

\section{References}

1. Greenberg M. Handbook of Neurosurgery. 8th ed. New York (NY): Thieme Medical Publisher; 2016.

2. Wang B, Hasadsri L, Wang H. Abdominal cerebrospinal fluid pseudocyst mimicking full-term pregnancy. J Surg Case Rep 2012; 2012: 6.

3. Ghritlaharey RK, Budhwani KS, Shrivastava DK, Srivastava J. Ventriculoperitoneal shunt complications needing shunt revision in children: a review of 5 years of experience with 48 revisions. Afr J Paediatr Surg 2012; 9: 32-39.

4. Shah H, Shah J, Mittal D. Abdominal cerebrospinal fluid pseudocysts in children: A complication of ventriculoperitoneal shunt. IJNS 2015; 4: 49-51.

5. Sebastian M, Sebastian A, Sroczyński M, Rudnicki J. Laparoscopic management of abdominal pseudocyst following ventriculoperitoneal shunt implantation in hydrocephalus. Videosurgery and Other Miniinvasive Techniques 2018; 13: 260.

6. Anwar R., Sadek A, Vajramani G. Abdominal pseudocyst: a rare complication of ventriculoperitoneal shunting. Pract Neurol 2017; 17: 212-213.

7. Salomão J, Leibinger R. Abdominal Pseudocysts Complicating CSF Shunting in Infants and Children. Pediatr Neurosurg. 1999; 31: 274-278.

8. Mobley LW 3rd, Doran S, Hellbusch L. Abdominal Pseudocyst: Predisposing Factors and Treatment Algorithm. Pediatr Neurosurg. 2005; 41: 77-83.

9. Hahn Y, Engelhard H, McLone D. Abdominal CSF Pseudocyst. Pediatr Neurosurg. 1985; 12: 75-79.

10. Popa F, Grigorean VT, Onose G, Popescu M, Strambu V, Sandu AM. Laparoscopic treatment of abdominal complications following ventriculoperitoneal shunt. J Med Life. 2009; 2: 426-436. 